\title{
ESTUDO DA ACURÁCIA NA AVALIAÇÃO DOS PÓLIPOS ENTRE A COLONOSCOPIA CONVENCIONAL, CROMOSCOPIA COM NARROW-BAND IMAGING E CROMOSCOPIA COM ÍNDIGO CARMIN
}

\author{
ACURACY STUDY ON POLYPE EVALUATION BETWEEN \\ CONVENTIONAL COLONOSCOPY, NARROW-BAND IMAGING \\ CHROMOSCOPY AND CARMIN INDIGO CHROMOSCOPY
}

\begin{abstract}
Anna Célia Arenas Rodrigues ${ }^{1 *}$, Gustavo Kurachi², Luciana Rodrigues de Meirelles ${ }^{3}$, Carlos Floriano de Morais ${ }^{4}$, Doryane Maria dos Reis Lima ${ }^{5}$, Mauro Willemann Bonatto $^{6}$

${ }^{1}$ Acadêmica do curso de Medicina da Faculdade Assis Gurgacz. ${ }^{2}$ Médico Gastroenterologista, Docente da Faculdade Assis Gurgacz, ${ }^{3}$ Médica Patologista, Doutora em Ciências Médicas. ${ }^{4}$ Médico Patologista, docente da Faculdade Assis Gurgacz e Doutor em Patologia. ${ }^{5}$ Médica Gastroenterologista, docente e preceptora da Residência em Cirurgia Geral da Fundação Hospitalar São Lucas e Doutora em Cirurgia. ${ }^{6}$ Médico Gastroenterologista, professor da disciplina de Gastroenterologia da Faculdade Assis Gurgacz e Doutor em Ciências da Saúde.
\end{abstract}

*Autor correspondente:anna.arenas@hotmail.com. https://orcid.org/0000-0002-5555-3513

\begin{abstract}
RESUMO
Introdução: As técnicas de cromoendoscopia aperfeiçoaram a acurácia na identificação de pólipos colorretais e contribuíram para a padronização de classificações. A classificação de Kudo avaliam as criptas intestinais através de corantes, enquanto que Japan NBI Expert Team avalia principalmente o padrão vascular da mucosa intestinal por meio da cromoendoscopia eletrônica. Ambos auxiliam na acurácia diagnóstica e rastreamento de câncer colorretal. Objetivo: Comparar a eficácia da colonoscopia convencional, cromoendoscopia com índigo e colonoscopia com Narrow-Band Imaging na acurácia diagnóstica de pólipos colorretais neoplásicos e não-neoplásicos. Materiais e métodos: Foram realizadas 119 polipectomias nos pólipos colorretais pequenos em centro especializado. As imagens dos pólipos foram avaliadas por um médico endoscopista experiente, que avaliou os padrões vasculares e superfícies dos pólipos de acordo com as classificações de Kudo e Japan NBI Expert Team, conforme o método utilizado (corante, Narrow-Band Imaging ou dedução em colonoscopia convencional). Os resultados das classificações foram comparados com 0 exame histológico. Conclusão: A colonoscopia com índigo, a colonoscopia com NBI e a colonoscopia convencional possuem eficácia no diagnóstico endoscópico, sendo a cromoscopia com índigo a de maior acurácia. Todavia, o diagnóstico endoscópico não deve ser exclusivo, sendo complementado pela avaliação histológica.
\end{abstract}

Palavras-chave: Colonoscopia. Cromoendoscopia. Pólipos colorretais.

\begin{abstract}
Introduction: Chromoendoscopy techniques improved the accuracy in identifying colorectal polyps and contributed to the standardization of classifications. The Kudo classification assesses intestinal crypts through dyes, while Japan NBI Expert Team mainly evaluates the vascular pattern of the intestinal mucosa by electronic chromoendoscopy. Both aid in diagnostic accuracy and colorectal cancer screening.
\end{abstract}


Objective: To compare the efficacy of conventional colonoscopy, indigo chromoendoscopy and Narrow-Band Imaging colonoscopy in the diagnostic accuracy of neoplastic and non-neoplastic colorectal polyps. Materials and methods: 119 polypectomies were performed on small colorectal polyps in a specialized center. Polyp images were evaluated by an experienced endoscopist, who evaluated the vascular patterns and surfaces of the polyps according to the Kudo and Japan NBI Expert Team classifications, according to the method used (dye, Narrow-Band Imaging or conventional colonoscopy deduction). ). The results of the classifications were compared with the histological examination. Conclusion: Indigo colonoscopy, NBI colonoscopy and conventional colonoscopy have efficacy in endoscopic diagnosis, with indigo chromoscopy being the most accurate. However, the endoscopic diagnosis should not be exclusive, being complemented by the histological evaluation.

Keywords: Colonoscopy. Chromoendoscopy. Colorectal Polyps.

\section{INTRODUÇÃO}

Os pólipos colorretais são achados frequentes nos exames de colonoscopia, embora na maioria das vezes são benignos, uma parcela destes são detectados com displasia ou já malignizados, por isso a avaliação endoscópica e histológica são de suma importância para a classificação e melhor planejamento de conduta tanto na ressecção quanto no follow up (GAGO et al, 2017).

De acordo com a histologia, os pólipos são classificados em neoplásicos (adenomatosos e serrilhados) e não neoplásicos (hiperplásicos, inflamatórios e hamartomas)(LI et al., 2014). Os pólipos neoplásicos são lesões benignas com potencial de progressão a malignidade, portanto são precursoras de carcinomas, enquanto que os pólipos malignos correspondem ao carcinoma invasor (GAGO et al, 2017).

A principal etiologia do câncer colorretal (CCR) são os pólipos adenomatosos que correspondem a $70-80 \%$ dos casos, seguido de pólipos serrilhados (20-25\%). Estima-se que $5 \%$ dos pólipos adenomatosos apresentam displasia de alto grau, conferindo-lhe maior probabilidade de progressão a carcinoma em aproximadamente 10 anos(GAGO et al, 2017).

Os pólipos serrilhados são subdivididos em pólipos hiperplásicos $(\mathrm{PH})$, adenoma serrilhado séssil (ASS) com ou sem displasia e adenoma serrilhado tradicional (AST), sendo que os dois últimos são considerados precursores de câncer colorretal(REX et al., 2012).

A colonoscopia é de extrema importância na redução da morbimortalidade oriunda do CCR, visto que permite o rastreamento, a identificação das lesões precursoras do CCR e malignas, polipectomias e retirada do material para biópsia. Com o avanço tecnológico, os aparelhos endoscópicos obtiveram o aperfeiçoamento de imagens, incorporando técnicas de magnificação e também de cromoendoscopia, mediante a aplicação de corantes ou eletrônica, viabilizando a visualização da mucosa intestinal, vascularização e a classificação dos pólipos com base no padrão mucoso, na qual presume a histologia dos pólipos (USSUI e WALLACE, 2012; VLEUGELS et al., 2017).

A cromoendoscopia convencional requer o uso de corantes, como índigo carmin e azul de metileno, assegurando as diferenciações dos pólipos a partir da visualização da mucosa intestinal, o que viabilizou a visualização de lesões diminutas, sésseis e a avaliação dos padrões das criptas. Essa técnica é limitada para a avaliação do padrão vascular, dado que os corantes dificultam a visualização dos 
vasos. Ao passo que o NBI, técnica de cromoendoscopia eletrônica, é um método endoscópico de aprimoramento de imagem que dispõe de filtros de luz a fim de aperfeiçoar as particularidades da mucosa e principalmente, o padrão vascular devido a absorção ótica da luz pela hemoglobina. Esta técnica apresenta boa equivalência com a histologia dos pólipos propiciando exatidão diagnóstica, diferenciação de lesões neoplásicas e não-neoplásica e auxílio na determinação de características neoplásica. Os benefícios do NBI refere-se a agilidade da utilização em razão da fácil ativação ao pressionar o interruptor presente no aparelho (alterna colonoscopia convencional e cromoendoscopia digital) e a capacidade de avaliar igualmente todo campo, ao contrário da cromoendoscopia com corante (J. J. W. TISCHENDORF, H. E. WASMUTH, A. KOCH, 2007; SANO et al., 2016; SUMIMOTO et al., 2016; TOGASHI et al., 2009; VLEUGELS et al., 2017; WADA et al., 2012; YOUNG PARK et al., 2008).

As classificações são ferramentas que auxiliam durante a colonoscopia para a distinção de pólipos com alta capacidade de evolução a carcinomas. A classificação de Kudo avaliam os padrões da mucosa intestinal, através da cromoendoscopia com uso de corantes que auxiliam na identificação de pólipos não neoplásicos (Tipo I e II), adenomatosos ( Tipo IIIL, IIls e IV) e malignos (Tipo V)(TISCHENDORF; WASMUTH; KOCH, 2007; KUDO, 1993; VLEUGELS et al., 2017). É indispensável à avaliação histológica em pólipos do Tipo II, visto que neles estão inclusos os pólipos serrilhados, precursores do CCR. (AVERBACH et al., 2003; KIMURA et al., 2012; KUDO, 1993; GAGO et al, 2017; VLEUGELS et al., 2017). Em contrapartida, o JNET é uma classificação para o Narrow Band Imaging System (NBI) capaz de avaliar o padrão das mucosa e principalmente o padrão vascular, sem a utilização de corantes (VLEUGELS et al., 2017; WADA et al., 2012), sua utilidade é semelhante a classificação de Kudo no sentido de predizer a histologia dos pólipos, subdividindo-se em tipo 1 (pólipos hiperplásicos e adenomas serrilhado séssil), tipo 2A (neoplasia intramucosa de baixo grau de displasia), tipo 2B (neoplasia intramucosa com alto grau de displasia ou câncer invasivo submucoso superficial) e tipo 3 (câncer invasivo submucoso profundo) (DJINBACHIAN; DUBÉ; VON RENTELN, 2019; KOMEDA et al., 2017).

Nenhum sistema de classificação consegue diferenciar adenomas com displasia de alto ou baixo grau e a diferenciação entre os pólipos sesseis serrilhados e SSA (DJINBACHIAN; DUBÉ; VON RENTELN, 2019). Ademais, estudos demonstram baixa equivalência entre patologistas na avaliação de pólipos SSA, assim como na diferenciação de PH e SSA (KIMURA et al., 2012).

O objetivo desse estudo prospectivo descritivo foi comparar a correlação do diagnóstico endoscópico da colonoscopia convencional, cromoendoscopia eletrônica (NBI) e cromoendoscopia com índigo com o diagnóstico histológico na determinação de pólipos colorretais neoplásicos e não-neoplásicos pequenos, menores que $10 \mathrm{~mm}$, com o auxílio das classificações de Kudo e JNET.

\section{METODOLOGIA}

Este estudo prospectivo e descritivo foi aprovado pelo Comitê de Ética em Pesquisa com Seres Humano (CEP) da Fundação Assis Gurgacz e todos os pacientes assinaram o Termo de Consentimento Livre e Esclarecido, autorizando a participação na pesquisa. Foram realizadas polipectomias em 67 pacientes submetidos à colonoscopia em centro especializado de endoscopia, totalizando uma amostra de 119 pólipos colorretais no período de Fevereiro de 2019 a Junho de 2019. 
Foram selecionadas as imagens dos 119 pólipos colorretais realizadas durante a colonoscopia e um endoscopia experiente, sem acesso aos dados histológicos, avaliou e classificou os pólipos colorretais de acordo com a classificação de Kudo, JNET ou dedução da superfície dos pólipos, conforme o método utilizado. Os pólipos corados com índigo carmin 0,4\% foram classificados em Kudo totalizando 22 pólipos, avaliados por aparelhos Olympus CF- Q145L, CF-Q150, CF-Q150L e CF- Q160ZL, enquanto que 33 pólipos foram submetidos à colonoscopia convencional, avaliados por aparelhos convencionais, e classificados de acordo com a dedução do endoscopista quanto a superfície dos pólipos. As colonoscopias realizadas com NBI e magnificação (Olympus CF- Q160ZL, CF- H180AL, CF-H170L) foram classificadas em JNET totalizando 64 pólipos. A técnica de magnificação não foi aplicada em pólipos corados com índigo carmin e os submetidos à colonoscopia convencional.

Posteriormente, foram analisadas as classificações realizadas pelo endoscopista nos três diferentes tipos de colonoscopias (convencional, com NBI e cromoscopia com índigo), com intuito de avaliar a eficácia das técnicas e classificações na acurácia diagnóstica. As classificações foram comparadas ao laudo histológico e os dados obtidos foram tabulados em planilhas do programa Microsoft Excelß .Todas as análises foram realizadas no software R.(R FOUNDATION FOR STATISTICAL COMPUTING, 2019)

Utilizou-se medidas de acurácia diagnóstica para discutir a eficácia das classificações realizadas pelo médico, sendo estas: especificidade $(e)$, sensibilidade $(s)$, valores preditivos positivos $(V P P)$ e negativos $(V P N)$, prevalência real $(P R)$ e estimada $(P E)$, acurácia $(a)$ e classificação incorreta $(C I)$.

As análises foram realizadas para cada tipo de colonoscopia, considerando-se "adenoma presente" para os indivíduos neoplásicos (Tipo III-L, III-s, IV e V da classificação de Kudo; Tipo 2A, 2B e 3 de JNET), e "adenoma ausente" para àqueles não-neoplásicos (Tipo I e II da classificação de Kudo; Tipo 1 de JNET). O teste foi considerado "positivo" quando a classificação do médico corroborou com o laudo histológico, e "negativo" caso contrário. Os pólipos colorretais serrilhados são considerados neoplásicos, todavia a classificação de Kudo não insere estes pólipos em sua classificação, tendo em vista que isto é um achado novo em relação a data de elaboração desta classificação. Em contrapartida, a classificação de JNET insere pólipos serrilhados sésseis no Tipo 1, juntamente com os hiperplásicos. Por esta razão, os pólipos designados como serrilhados na análise da histopatologia foram considerados neoplásicos, deste modo os pólipos serrilhados classificados em JNET Tipo 1 foram considerados como subdiagnosticados.

Quadro 1 - Validade de um teste diagnóstico

\begin{tabular}{|c|c|c|c|}
\hline & \multicolumn{2}{|c|}{ ADENOMA } \\
\hline & & PRESENTE & AUSENTE \\
\hline \multirow{2}{*}{ TESTE } & POSITIVO & $\begin{array}{c}\text { Verdadeiro } \\
\text { Positivo (VP) }\end{array}$ & $\begin{array}{r}\text { Falso- } \\
\text { Positivo (FP) }\end{array}$ \\
\hline & NEGATIVO & $\begin{array}{r}\text { Falso- } \\
\text { Negativo (FN) }\end{array}$ & $\begin{array}{l}\text { Verdadeiro } \\
\text { Negativo (VP) }\end{array}$ \\
\hline
\end{tabular}

$\mathrm{Na}$ avaliação de um teste diagnóstico existem 4 interpretações possíveis para o resultado do teste: duas em que o teste está correto e duas em que está incorreto. O teste está correto quando ele é positivo na presença de adenoma (resultados verdadeiros positivos), ou negativo na ausência de adenoma (resultados verdadeiros negativos). Por outro lado, o teste está incorreto quando ele é positivo na ausência de adenoma (falso positivo), ou negativo quando o adenoma está presente (falso negativo) (quadro 1). 


\section{RESULTADOS E DISCUSSÃO}

A validade do teste diagnostico em relação a colonoscopia convencional (quadro 2) sem técnicas de cromoscopia e magnificação, o endoscopista obteve especificidade (e) de $72,72 \%$ e sensibilidade (s) de $68,18 \%$, demonstrando ter sido mais eficiente em diagnosticar pólipos neoplásicos do que os não-neoplásicos. O valor preditivo positivo (VPP) foi de $83,33 \%$ e valor preditivo negativo (VPN) de $53,33 \%$. A prevalência real (PR) $(66,67 \%)$ e estimada (PE) $(30,30 \%)$ tiveram uma diferença de pouco mais de $36 \%$ entre si. A acurácia (a) foi de $69,69 \%$ e a classificação incorreta (Cl) foi de $30,30 \%$.

Quadro 2 - Validade de um teste de diagnóstico e medidas de acurácia considerando pacientes avaliados em Colonoscopia Convencional

\begin{tabular}{|c|c|c|c|}
\hline \multirow{2}{*}{$\begin{array}{l}\text { Colonoscopia } \\
\text { Convencional } \\
\text { Diagnóstico }\end{array}$} & \multirow[b]{2}{*}{$\begin{array}{c}\text { Positivo } \\
\text { Negativo }\end{array}$} & \multicolumn{2}{|c|}{ Neoplásico } \\
\hline & & $\begin{array}{c}\text { Presente } \\
15 \text { (VP) } \\
7 \text { (FN) } \\
\end{array}$ & $\begin{array}{c}\text { Ausente } \\
3(\mathrm{FP}) \\
8(\mathrm{VN}) \\
\end{array}$ \\
\hline $\begin{array}{c}\text { Parâmetro } \\
\text { E } \\
\text { S } \\
\text { PR } \\
\text { PE } \\
\text { VPP } \\
\text { VPN } \\
\text { A } \\
\text { Cl }\end{array}$ & \begin{tabular}{c}
\multicolumn{2}{c}{ Valor } \\
$(\%)$ \\
72,72 \\
68,18 \\
66,67 \\
30,30 \\
83,33 \\
53,33 \\
69,69 \\
30,30
\end{tabular} & & \\
\hline
\end{tabular}

VP: verdadeiro positivo; FP: falso positivo; FN: falso negativo; VN: verdadeiro negativo; e: especificidade; s: sensibilidade; PR: prevalência real; PE: prevalência estimada; VPP: valor preditivo positivo; VPN: valor preditivo negativo; a: acurácia; $\mathrm{Cl}$ : classificação incorreta.

Com a utilização da Cromoscopia com índigo, o endoscopista obteve especificidade (e) de 100,00\% e sensibilidade (s) de $61,54 \%$. O valor preditivo positivo (VPP) foi de $100,00 \%$ e valor preditivo negativo (VPN) de $64,29 \%$. A prevalência real (PR) $(59,09 \%)$ e estimada (PE) $(22,73 \%)$ tiveram uma diferença superior a $30 \%$ entre si. A acurácia (a) foi de $77,27 \%$ e a classificação incorreta (Cl) foi de $22,73 \%$ (quadro 3).

Em relação à colonoscopia com $\mathrm{NBI}$, o endoscopista obteve especificidade (e) de $48,00 \%$ e sensibilidade (s) de $79,49 \%$, indicando foi mais eficaz em diagnosticar os pólipos neoplásicos do que os não-neoplásicos. O valor preditivo positivo (VPP) foi de $70,45 \%$ e valor preditivo negativo (VPN) de $60,00 \%$. A diferença entre a prevalência real (PR) $(60,94 \%)$ e estimada (PE) $(32,81 \%)$ foi cerca de $30 \%$. A acurácia (a) foi de $67,19 \%$ e a classificação incorreta (Cl) foi de $32,81 \%$ (quadro 4 ).

Diante o exposto, a colonoscopia com NBI, a cromoscopia com índigo e a colonoscopia convencional obtiveram forte concordância do diagnóstico endoscópico com o diagnóstico histológico, sendo que a cromoscopia com índigo obteve o melhor desempenho (acurácia 77,27\%), mesmo sem a utilização de técnicas de magnificação. Isso se deve a alta capacidade da técnica em identificar os pólipos nãoneoplásicos, visto que obteve especificidade de $100 \%$, isto é, todos os pólipos nãoneoplásicos foram identificados. 
Quadro 3 - Validade de um teste de diagnóstico e medidas de acurácia na Cromoscopia com Índigo

\begin{tabular}{|cccc|}
\hline Com Índigo & & \multicolumn{2}{c|}{ Neoplásico } \\
\cline { 3 - 4 } Diagnóstico & Positivo & $8(\mathrm{VP})$ & 0 (FP) \\
& Negativo & $5(\mathrm{FN})$ & $9(\mathrm{VN})$ \\
\hline \multirow{3}{*}{ Valor } & \\
Parâmetros & $(\%)$ & & \\
E & 100,00 & \\
S & 61,54 & \\
PR & 59,09 & \\
PE & 22,73 & \\
VPP & 100,00 & \\
VPN & 64,29 & \\
A & 77,27 & \\
Cl & 22,73 & \\
\hline
\end{tabular}

VP: verdadeiro positivo; FP: falso positivo; FN: falso negativo; VN: verdadeiro negativo; e: especificidade; s: sensibilidade; PR: prevalência real; PE: prevalência estimada; VPP: valor preditivo positivo; VPN: valor preditivo negativo; a: acurácia; Cl: classificação incorreta.

Embora a técnica de NBI não tenha alcançado o melhor índice de acertos no diagnóstico endoscópico dos pólipos colorretais (acurácia 67,19\%), esta foi a mais sensível dentre as outras, ou seja, com maior capacidade de detectar os pólipos com potenciais neoplásicos, fato que pode estar relacionado com a capacidade do aparelho evidenciar o padrão vascular das lesões neoplásicas permitindo o diagnóstico. Além disso, foi a técnica que mais se aproximou da prevalência real. Entretanto, os índices de correlação endoscópica com o laudo histológico foi o menor na avaliação de pólipos não-neoplásicos, supervalorizando os pólipos.

Quadro 4 - Validade de um teste de diagnóstico e medidas de acurácia em colonoscopia com NBI

\begin{tabular}{|cccc|}
\hline Colonoscopia & & \multicolumn{2}{c|}{ Neoplásico } \\
\cline { 3 - 4 } com NBI & & Presente & Ausente \\
Diagnóstico & Positivo & 31 (VP) & 13 (FP) \\
& Negativo & $8(\mathrm{FN})$ & 12 (VN) \\
\hline Parâmetros & $(\%)$ & \\
e & 48,00 & \\
S & 79,49 & \\
PR & 60,94 & \\
PE & 32,81 & \\
VPP & 70,45 & \\
VPN & 60,00 & \\
a & 67,19 & \\
Cl & 32,81 & \\
\hline
\end{tabular}

VP: verdadeiro positivo; FP: falso positivo; FN: falso negativo; VN: verdadeiro negativo; e: especificidade; s: sensibilidade; PR: prevalência real; PE: prevalência estimada; VPP: valor preditivo positivo; VPN: valor preditivo negativo; a: acurácia; $\mathrm{Cl}$ : classificação incorreta.

A colonoscopia convencional, assim como as outras, também indicou boa correlação com o diagnóstico histológico. No entanto, demonstrou-se superior na identificação de pólipos neoplásicos (sensibilidade 68,18\%) em relação a cromoscopia com índigo (sensibilidade 61,54\%). 
Assim, neste estudo a cromoendoscopia com índigo carmin obteve resultados superiores aos outros métodos em relação a acurácia, concordando com os estudos que demonstram a superioridade da cromoendoscopia com índigo e também da colonoscopia com uso de NBI na acurácia diagnóstica (CHANG et al., 2009; CHIU et al., 2007). Ainda, o auxílio das classificações de Kudo e JNET foram eficientes, tendo em vista a forte correlação diagnósticas que os métodos obtiveram. Todavia, o estudo possui viés a respeito da ausência de magnificação em todos pólipos colorretais, o que poderia demonstrar de forma mais satisfatória a vantagem de um método em relação aos outros.

\section{CONSIDERAÇÕES FINAIS}

A colonoscopia com índigo carmin, a colonoscopia com NBI e a colonoscopia convencional possuem eficácia no diagnóstico endoscópico, mesmo sem a utilização de magnificação na colonoscopia convencional e na colonoscopia com índigo, sendo a colonoscopia com índigo a de melhor acurácia tendo em vista sua alta capacidade de identificar pólipos não-neoplásicos. Contudo, a técnica de NBI é a mais eficaz na identificação de pólipos neoplásicos, o que é de importância clínica visto que estes pólipos possuem potencial de evolução para o CCR. Ainda assim, o diagnóstico endoscópico não é superior ao histológico, sendo este necessário para a determinação histológica.

Este estudo pode auxiliar na decisão da utilização de técnicas de remoção de pólipos (à frio, com pinça, com alça ou técnicas de mucosectomia) para a obtenção de um material completo.

\section{REFERÊNCIAS}

CHANG, C. C. et al. Comparative study of conventional colonoscopy, magnifying chromoendoscopy, and magnifying narrow-band imaging systems in the differential diagnosis of small colonic polyps between trainee and experienced endoscopist. International Journal of Colorectal Disease, [s. I.], v. 24, n. 12, p. 1413-1419, 2009. DOI 10.1007/s00384-009-0760-9

CHIU, H. M. et al. A prospective comparative study of narrow-band imaging, chromoendoscopy, and conventional colonoscopy in the diagnosis of colorectal neoplasia. Gut, [s. I.], v. 56, n. 3, p. 373-379, 2007. DOI: 10.1136/gut.2006.099614

DJINBACHIAN, R.; DUBÉ, A.-J.; VON RENTELN, D. Optical Diagnosis of Colorectal Polyps: Recent Developments. Current Treatment Options in Gastroenterology, [s. I.], v. 17, n. 1, p. 99-114, 2019. DOI: 10.1007/s11938-019-00220-x

J. J. W. TISCHENDORF, H. E. WASMUTH, A. KOCH, Et al. Value of magnifying chromoendoscopy and narrow band imaging (NBI) in classifying colorectal polyps: a prospective controlled study. Endoscopy, [s. I.], v. 39, p. 1092-1096, 2007. DOI: $10.1055 / \mathrm{s}$

KOMEDA, Y. et al. Magnifying Narrow Band Imaging (NBI) for the Diagnosis of Localized Colorectal Lesions Using the Japan NBI Expert Team (JNET) Classification. Oncology (Switzerland), [s. I.], v. 93, n. 1, p. 49-54, 2017.DOI 10.1159/000481230 
KUDO, S. Endoscopic Mucosal Resection of Flat and Depressed Types of Early Colorectal Cancer. Endoscopy, [s. I.], v. 25, p. 455-461, 1993.

LI, M. et al. Kudo's pit pattern classification for colorectal neoplasms: A meta-analysis. World Journal of Gastroenterology, [s. I.], v. 20, n. 35, p. 12649-12656, 2014. DOI: 10.3748/wjg.v20.i35.12649

R FOUNDATION FOR STATISTICAL COMPUTING. R Development Core Team. R: A language and environment for statistical computingVienna, 2019.

REX, D. K. et al. Serrated Lesions of the Colorectum: Review and Recommendations From an Expert Panel. American Journal of Gastroenterology, [s. I.], v. 107, n. 9, p. 1315-1329, 2012. DOI: 10.1038/ajg.2012.161

SANO, Y. et al. Narrow-band imaging (NBI) magnifying endoscopic classification of colorectal tumors proposed by the Japan NBI Expert Team. Digestive Endoscopy, [s. I.], p. 1-8, 2016. DOI: 10.1016/j.gie.2016.07.035

SUMIMOTO, K. et al. Clinical impact and characteristics of the narrow-band imaging magnifying endoscopic classification of colorectal tumors proposed by the Japan NBI Expert Team. Gastrointestinal Endoscopy, [s. I.], 2016. http://dx.doi.org/10.1016/j.gie.2016.07.035

T. GAGO; AM. VAZ; P. QUEIRÓS; J. ROSEIRA; AC. CUNHA; AC. ARAÚJO; A. RAMOS; P. CALDEIRA; H. GUERREIRO. Pólipos colo-rectais e sua importância clínica. Revista Portuguesa De Coloproctologia, [s. I.], v. Mai/Out, p. 50-60, 2017. DOI: $10.1080 / 00365520801935442$

TOGASHI, K. et al. A comparison of conventional endoscopy, chromoendoscopy, and the optimal-band imaging system for the differentiation of neoplastic and nonneoplastic colonic polyps. Gastrointestinal Endoscopy, [s. I.], v. 69, n. 3 SUPPL., p. 734-741, 2009. http://dx.doi.org/10.1016/j.gie.2008.10.063

USSUI, V. M.; WALLACE, M. B. Imaged-enhanced Technologies for Colorectal Polyp Detection and Classification. The American Journal of Gastroenterology, [s. I.], v. 107, n. 4, p. 551-553, 2012. Disponível em: <http://dx.doi.org/10.1038/ajg.2012.15>

VLEUGELS, J. L. A. et al. Morphological classifications of gastrointestinal lesions. Best Practice \& Research Clinical Gastroenterology, [s. I.], v. 31, p. 359-367, 2017. DOI: 10.1016/j.bpg.2017.05.005

WADA, Y. et al. Diagnostic accuracy of pit pattern and vascular pattern analyses in colorectal lesions. Gastroenterological Endoscopy, [s. I.], v. 54, n. 6, p. 1873-1882, 2012. DOI: $10.1111 / \mathrm{j} .1443-1661.2010 .00983 . x$

YOUNG PARK, S. et al. Efficacy of chromoendoscopy with indigocarmine for the detection of ascending colon and cecum lesions. Scandinavian Journal of Gastroenterology, [s. I.], $\quad$ v. $43, \quad$ n. $\quad 7, \quad$ p. $878-885,2008 . \quad$ DOI: 10.1080/00365520801935442 